\title{
REGULAR FRACTIONAL FACTORIAL DESIGNS WITH MINIMUM ABERRATION AND MAXIMUM ESTIMATION CAPACITY
}

\author{
By Ching-Shui Cheng ${ }^{1}$ and Rahul MukerJee ${ }^{2}$ \\ University of California, Berkeley and \\ Indian Institute of Management
}

\begin{abstract}
Using the approach of finite projective geometry, we make a systematic study of estimation capacity, a criterion of model robustness, under the absence of interactions involving three or more factors. Some general results, providing designs with maximum estimation capacity, are obtained. In particular, for two-level factorials, it is seen that constructing a design with maximum estimation capacity calls for choosing points from a finite projective geometry such that the number of lines is maximized and the distribution of these lines among the chosen points is as uniform as possible. We also explore the connection with minimum aberration designs under which the sizes of the alias sets of two-factor interactions which are not aliased with main effects are the most uniform possible.
\end{abstract}

1. Introduction. The notion of minimum aberration, introduced by Fries and Hunter (1980), is a commonly used criterion for choosing good fractional factorial designs. It is a refinement of the resolution criterion of Box and Hunter (1961). Construction of regular fractional factorial designs with minimum aberration has been considered, for example, by Franklin (1984), Chen and Wu (1991), Chen (1992), Chen, Sun and Wu (1993), Tang and Wu (1996) and Suen, Chen and Wu (1997). Chen and Hedayat (1996) considered designs with weak minimum aberration. In an attempt to provide statistical justification for the primarily combinatorial criterion of minimum aberration, Cheng, Steinberg and Sun (1998) looked at the criterion of estimation capacity [Sun (1993)], a measure of the capability of a design to handle and estimate different potential models involving interactions.

The present work aims at studying in a more systematic manner the issue of estimation capacity and the alias patterns of two-factor interactions under minimum aberration designs. As in most practical situations, we consider a situation where the main effects are of primary interest and, under the absence of interactions involving three or more factors, interest lies in having

Received October 1996; revised March 1998.

${ }^{1}$ Supported in part by NSF Grant DMS-94-04477 and NSA Grant MDA-904-95-1-1064.

${ }^{2}$ Supported in part by a grant from the Centre of Management and Development Studies, Indian Institute of Management, Calcutta.

AMS 1991 subject classification. Primary 62K15.

Key words and phrases. Estimation capacity, projective geometry, resolution, upper weak majorization, weak minimum aberration. 
as much information on the two-factor interaction pencils as possible. Unlike Cheng, Steinberg and Sun (1998), who considered only 16- and 32-run two-level factorials via a computer search, we attempt to develop the underlying theory using a finite projective geometric approach. This enables us to obtain general theoretical results, some of which pertain even to $s$-level factorials in general. In particular, it is seen in Section 2 that complementary designs play a crucial role in this context and greatly facilitate the study of estimation capacity in the practically important nearly saturated case. Results for two-level designs are reported in Section 3, while Section 4 considers designs with more than two levels. Our results provide a justification of minimum aberration as a good surrogate for the statistically more meaningful criterion of maximum estimation capacity.

Now we present some preliminary material including definitions and notations. Consider the setup of an $s^{n}$ factorial experiment where $s(\geq 2)$ is a prime or a prime power. A typical pencil belonging to a factorial effect is an $n \times 1$ nonnull vector $\mathbf{b}$, with elements from $G F(s)$, the finite field with $s$ elements. For $\lambda(\neq 0) \in G F(s), \mathbf{b}$ and $\lambda \mathbf{b}$ represent the same pencil. A pencil b represents a main effect if it has exactly one nonzero element, and it represents a two-factor interaction if there are exactly two nonzero elements. We shall consider regular $s^{n-k}$ designs of resolution at least three and call them resolution $\mathrm{III}^{+}$designs. It is well known [Bose (1947)] that such designs arise from the finite projective geometry $P G(n-k-1, s)$ as follows. Let $\boldsymbol{\alpha}_{1}, \ldots, \boldsymbol{\alpha}_{g}$ be the distinct points of $P G(n-k-1, s)$, where $g=\left(s^{n-k}-1\right)$ / $(s-1), T$ be any $n$-subset of $G=\{1, \ldots, g\}$ and $\mathbf{V}_{T}$ be the $(n-k) \times n$ matrix with columns $\boldsymbol{\alpha}_{i}, i \in T$. Then the design given by $T$ consists of the $s^{n-k}$ level combinations belonging to the row space of $\mathbf{V}_{T}$ (called a principal fraction) or any coset thereof in the finite Euclidean geometry $E G(n, s)$. These $s^{n-k}$ level combinations are distinct provided $\mathbf{V}_{T}$ has full row rank, a condition which is assumed hereafter. Also, to avoid trivialities, we suppose $n<g$. Then $f \equiv g-n$ is positive.

A pencil $\mathbf{b}$ appears in the defining equation of the design represented by $T$ if and only if $\mathbf{V}_{T} \mathbf{b}=\mathbf{0}$. For any $s^{n-k}$ fractional factorial design, let $A_{i}$ be the number of $i$-factor interaction pencils appearing in the defining equation. Then the resolution is the smallest $i$ such that $A_{i}>0$, and the criterion of minimum aberration successively minimizes the $A_{i}$ 's in the increasing order of $i$. Let $r_{\max }$ denote the maximum possible resolution of a design for given $s$, $n$ and $k$. If a design has resolution $r_{\max }$ and minimizes $A_{r_{\max }}$ among all the designs of resolution $r_{\max }$, then it is said to have weak minimum aberration [Chen and Hedayat (1996)].

Two distinct pencils $\mathbf{b}_{1}$ and $\mathbf{b}_{2}$, neither of which appears in the defining equation, are aliased with each other if and only if $\mathbf{V}_{T} \mathbf{b}_{1}$ and $\mathbf{V}_{T} \mathbf{b}_{2}$ are proportional to the same point of the projective geometry. Since $\operatorname{rank}\left(\mathbf{V}_{T}\right)=$ $n-k$, there are a total of $g$ alias sets, where each alias set corresponds to $s-1$ degrees of freedom. Under a resolution $\mathrm{III}^{+}$design, no main effect pencil appears in its defining equation, and no two distinct main effect pencils are aliased with each other. Thus there are $n$ distinct alias sets, each 
of which contains one main effect pencil. For $1 \leq j \leq f$, let $m_{j}(T)$ be the number of two-factor interaction pencils in the $j$ th of the remaining $f$ $(=g-n)$ alias sets. Define the $f \times 1$ vector $\mathbf{m}(T)=\left(m_{1}(T), \ldots, m_{f}(T)\right)^{\prime}$. Note that the nonzero elements among $m_{1}(T), \ldots, m_{f}(T)$ are the sizes of the alias sets of two-factor interactions which are not aliased with main effects.

Note that there are $\left(\begin{array}{l}n \\ 2\end{array}\right)(s-1)(=\nu$, say) distinct two-factor interaction pencils. For $1 \leq u \leq \nu$, let $E_{u}(T)$ be the number of models containing all the main effects and $u$ two-factor interaction pencils which can be estimated by the design T. Analogous to equation (3.1) of Cheng, Steinberg and Sun (1998),

$$
E_{u}(T)= \begin{cases}\sum_{1 \leq i_{1}<\cdots<i_{u} \leq f} \prod_{j=1}^{u} m_{i_{j}}(T), & \text { if } u \leq f, \\ 0, & \text { otherwise. }\end{cases}
$$

It is desirable to have the quantities $E_{u}(T)$ as large as possible. A design which maximizes $E_{u}(T)$ for all $u(1 \leq u \leq \nu)$ is said to have maximum estimation capacity. For any two designs $T_{1}$ and $T_{2}$, we say that $T_{1}$ dominates $T_{2}$ (with respect to estimation capacity) if $E_{u}\left(T_{1}\right) \geq E_{u}\left(T_{2}\right)$ for all $u$, with strict inequality for some $u$. As in Cheng, Steinberg and Sun (1998), we have the following helpful lemma which can be proved by using (1.1) via a Schur concavity argument.

Lemma 1.1. Given two designs $T_{1}$ and $T_{2}$, if $\mathbf{m}\left(T_{1}\right)$ is upper weakly majorized by $\mathbf{m}\left(T_{2}\right)$ and not obtainable from $\mathbf{m}\left(T_{2}\right)$ by permuting its elements, then $T_{1}$ dominates $T_{2}$.

Therefore, a design has large estimation capacity if $\sum_{i=1}^{f} m_{i}(T)$ is large and the $m_{i}(T)$ 's are as equal as possible. Note that $\sum_{i=1}^{f} m_{i}(T)$ is equal to the total number of two-factor interaction pencils which are not aliased with main effects. A design with (weak) minimum aberration must maximize this quantity. But this is only part of what is required for a design to be "optimal" in the sense of Lemma 1.1.

2. Connection with the complementary design. Note that for each pencil $\mathbf{b}$ belonging to the same alias set of $T, \mathbf{V}_{T} \mathbf{b}$ is proportional to the same point of $P G(n-k-1, s)$. This establishes a one-to-one correspondence between the $g$ alias sets and the $g$ points in $P G(n-k-1, s)$. If an alias set does not contain any main effect, then the corresponding point must be the same as $\alpha_{i}$ for some $i \in \bar{T}$, where $\bar{T}$ is the complement of $T$ in $G=\{1, \ldots, g\}$. Hence defining for each $i \in \bar{T}, h_{i}(\bar{T})$ as the number of distinct two-factor interaction pencils $\mathbf{b}$ such that $\mathbf{V}_{T} \mathbf{b}$ is proportional to $\boldsymbol{\alpha}_{i}$, and $\mathbf{h}(\bar{T})$ as an $f \times 1$ vector with elements $h_{i}(\bar{T}), i \in \bar{T}$, the following is evident.

LEMMA 2.1. The vector $\mathbf{m}(T)$ can be obtained from $\mathbf{h}(\bar{T})$ by permuting the elements of the latter. 
We now introduce some more notation. For any $\lambda(\neq 0) \in G F(s)$ and any three distinct members $i, j, r$ of $G$, define the indicators

$$
\begin{aligned}
\xi_{i j r}(\lambda) & = \begin{cases}1, & \text { if } \boldsymbol{\alpha}_{j}+\lambda \boldsymbol{\alpha}_{r} \text { is proportional to } \boldsymbol{\alpha}_{i}, \\
0, & \text { otherwise }\end{cases} \\
\theta_{i j r} & = \begin{cases}1, & \text { if } \boldsymbol{\alpha}_{i}, \boldsymbol{\alpha}_{j} \text { and } \boldsymbol{\alpha}_{r} \text { are linearly dependent } \\
0, & \text { otherwise }\end{cases}
\end{aligned}
$$

It is easy to see that for any distinct $i, j, r \in G$,

$$
\sum_{\lambda \neq 0} \xi_{i j r}(\lambda)=\theta_{i j r}
$$

and that for any fixed $i, j \in G, i \neq j$,

$$
\sum_{r \in G, r \neq i, j} \theta_{i j r}=s-1 .
$$

For each fixed $i \in \bar{T}$, define $\phi_{i}(\bar{T})$ as the number of linearly dependent triplets $\left\{\boldsymbol{\alpha}_{i}, \boldsymbol{\alpha}_{j}, \boldsymbol{\alpha}_{r}\right\}$ such that $i, j$ and $r$ are distinct members of $\bar{T}$ and $j<r$. Then the following lemma holds.

Lemma 2.2. For each $i \in \bar{T}, h_{i}(\bar{T})=\frac{1}{2}(s-1)(g-2 f+1)+\phi_{i}(\bar{T})$.

Proof. For any fixed $i \in \bar{T}$, let $\Delta_{1 i}(\bar{T}), \Delta_{2 i}(\bar{T}), \Delta_{3 i}, \Delta_{4 i}(\bar{T}), \Delta_{5 i}(\bar{T})$ and $\Delta_{6 i}(\bar{T})$ denote sums over $\theta_{i j r}$ with respect to $j$ and $r$, the range of summation being:

(i) $j \in T, r \in T, j<r$;

(ii) $j \in T, r \in T, j \neq r$;

(iii) $j \in G, r \in G, j \neq r, j \neq i, r \neq i$;

(iv) $j \in \bar{T}, r \in \bar{T}, j \neq r, j \neq i, r \neq i$;

(v) $j \in \bar{T}, r \in T, j \neq i$;

(vi) $j \in T, r \in \bar{T}, r \neq i$,

respectively. By (2.2) and the definition of $\phi_{i}(\bar{T})$, note that

$$
\Delta_{1 i}(\bar{T})=\frac{1}{2} \Delta_{2 i}(\bar{T}), \quad \Delta_{5 i}(\bar{T})=\Delta_{6 i}(\bar{T}), \quad \phi_{i}(\bar{T})=\frac{1}{2} \Delta_{4 i}(\bar{T})
$$

Recall that for each $i \in \bar{T}, h_{i}(\bar{T})$ is the number of distinct two-factor interaction pencils $\mathbf{b}$ such that $\mathbf{V}_{T} \mathbf{b}$ is proportional to $\boldsymbol{\alpha}_{i}$. Hence by (2.1), (2.3), (2.5) and the definition of $\mathbf{V}_{T}$,

$$
\begin{aligned}
h_{i}(\bar{T}) & =\sum_{j<r, j, r \in T} \sum_{\lambda \neq 0} \xi_{i j r}(\lambda)=\Delta_{1 i}(\bar{T})=\frac{1}{2} \Delta_{2 i}(\bar{T}) \\
& =\frac{1}{2}\left(\Delta_{3 i}-\Delta_{4 i}(\bar{T})-\Delta_{5 i}(\bar{T})-\Delta_{6 i}(\bar{T})\right) \\
& =\frac{1}{2}\left(\Delta_{3 i}-\Delta_{4 i}(\bar{T})-2 \Delta_{5 i}(\bar{T})\right) .
\end{aligned}
$$


Now for fixed $i, j \in \bar{T}, j \neq i$, by (2.4),

$$
\sum_{r \in T} \theta_{i j r}=C_{1}-C_{2}=s-1-C_{2}
$$

where $C_{1}$ and $C_{2}$ are the sums of $\theta_{i j r}$ over $r \in G, r \neq i, j$ and $r \in \bar{T}, r \neq i, j$, respectively. Summing (2.7) with respect to $j(j \in \bar{T}, j \neq i)$,

$$
\Delta_{5 i}(\bar{T})=(s-1)(f-1)-\Delta_{4 i}(\bar{T}) .
$$

Similarly, for fixed $i \in \bar{T}$, summing (2.4) with respect to $j(j \in G, j \neq i$ ),

$$
\Delta_{3 i}=(s-1)(g-1) .
$$

If one substitutes (2.8) and (2.9) in (2.6) and employs the last relation in (2.5), then the result follows.

Let $\phi(\bar{T})$ be an $f \times 1$ vector with elements $\phi_{i}(\bar{T}), i \in \bar{T}$. From Lemmas 1.1, 2.1 and 2.2, we have the following result.

THeOREm 2.1. Let $T_{1}$ and $T_{2}$ be two designs. If $\phi\left(\bar{T}_{1}\right)$ is upper weakly majorized by $\phi\left(\bar{T}_{2}\right)$ and not obtainable from $\phi\left(\bar{T}_{2}\right)$ by permuting its elements, then $T_{1}$ dominates $T_{2}$.

As will be seen later, Theorem 2.1 leads to further results on maximum estimation capacity. The advantage of Theorem 2.1 is that one needs to consider the set $\bar{T}$, of cardinality $f$, alone to obtain $\phi(\bar{T})$. Thus Lemma 2.2 and Theorem 2.1 facilitate the study of estimation capacity particularly for small $f$, a situation which corresponds to nearly saturated cases and can be of practical interest.

Two designs $T_{1}$ and $T_{2}$ will be said to be isomorphic to each other if there exists a nonsingular matrix $\Lambda$, of rank $n-k$ and defined over $G F(s)$, such that for every $i \in T_{1}, \Lambda \boldsymbol{\alpha}_{i}$ is proportional to some $\boldsymbol{\alpha}_{j}, j \in T_{2}$. Hence if $T_{1}$ and $T_{2}$ are isomorphic designs, then $\phi\left(\bar{T}_{1}\right)$ is obtainable by permuting the elements of $\phi\left(\bar{T}_{2}\right)$, so that $E_{u}\left(T_{1}\right)=E_{u}\left(T_{2}\right)$ for each $u$. Thus one needs to consider only nonisomorphic designs while investigating estimation capacity. In particular, for $f=1$ or 2 , all designs are isomorphic to one another, and consequently, for $n=g-1$ or $g-2$, all regular $s^{n-k}$ designs of resolution $\mathrm{III}^{+}$are equivalent with respect to estimation capacity. Since isomorphism also entails identical word-length patterns [see Tang and Wu (1996)], it follows that for $f=1$ or 2 , the criteria of minimum aberration and estimation capacity are trivially in perfect agreement. Hereafter, only the situation $f \geq 3$ will be considered.

Our studying of estimation capacity via consideration of complementary subsets and isomorphism is in the spirit of Tang and Wu (1996) and Suen, Chen and $\mathrm{Wu}$ (1997) who, for $s=2$ and general $s$, respectively, characterized minimum aberration designs in terms of complementary designs. Summing the identities in Lemma 2.2 over $i \in \bar{T}$, one gets their identity for the three-factor interaction pencils in the defining relation. Our problem, how- 
ever, is different from theirs. While they were concerned only with the defining equation, we have to take care of the aliasing pattern explicitly.

3. $2^{n-k}$ designs with maximum estimation capacity. Throughout this section, we consider the case $s=2$. For simplicity, hereafter, we shall not distinguish between $G$ and $P G(n-k-1,2)$. Each $T$ is considered as an $n$-subset of $P G(n-k-1,2)$, and $\bar{T}$ is the complement of $T$ in $P G(n-k-$ 1,2). Consequently, $\operatorname{rank}(T)$ and $\operatorname{rank}(\bar{T})$ are now well defined and $\operatorname{rank}(T)$ equals $\operatorname{rank}\left(\mathbf{V}_{T}\right)$. Three distinct points (vectors) of $P G(n-k-1,2)$ are linearly dependent if and only if their sum equals the null vector. Three such points constitute a line. Hence for any $f$-subset $\bar{T}$ of $P G(n-k-1,2)$ and any $1 \leq i \leq f, \phi_{i}(\bar{T})$ can be geometrically interpreted as the number of lines that pass through the $i$ th point of $\bar{T}$ and two other distinct points of $\bar{T}$. Then $\frac{1}{3} \sum_{i=1}^{f} \phi_{i}(\bar{T})$ is equal to the total number of lines contained in $\bar{T}$, and to maximize $\sum_{i=1}^{f} \phi_{i}(\bar{T})$ is the same as to maximize the number of lines contained in $\bar{T}$. Therefore, in view of Theorem 2.1, our problem is to choose $f$ points from $P G(n-k-1,2)$ containing the maximum number of lines, and these lines should be as uniformly distributed among the $f$ points as possible. Chen and Hedayat's (1996) work on weakly minimum aberration designs solves the problem of choosing $f$ points from $P G(n-k-1,2)$ which contain the most lines, but we also need the $\phi_{i}(\bar{T})$ 's to be as equal as possible.

Following Chen and Hedayat (1996), if $2^{w-1} \leq f<2^{w}(w \leq n-k)$, then an $f$-subset $\bar{T}$ of $P G(n-k-1,2)$ contains the maximum number of lines if and only if $\bar{T}=\mathscr{F} \backslash \mathscr{H}$, that is, the complement of $\mathscr{H}$ in $\mathscr{F}$, where $\mathscr{F}$ is any $(w-1)$-flat in $P G(n-k-1,2)$ and $\mathscr{H}$, a $\left(2^{w}-1-f\right)$-subset of $\mathscr{F}$, contains no line at all. Chen and Hedayat (1996) showed that it is always possible to find a $\left(2^{w}-1-f\right)$-subset $\mathscr{H}$ of $\mathscr{F}$ which contains no line. Here by a $(w-1)$ flat we mean the set of all the points which are linear combinations of $w$ linearly independent points in a projective geometry.

We have the following result on designs with maximum estimation capacity.

TheOREM 3.1. Suppose $2^{w-1} \leq f<2^{w}(w \leq n-k)$ and $\mathscr{F}$ is $a(w-1)$ flat. Furthermore, suppose there exists a $\left(2^{w}-1-f\right)$-subset $\mathscr{H}^{*}$ of $\mathscr{F}$ such that any three or four distinct points of $\mathscr{H}^{*}$ are linearly independent. Then the design given by $T^{*}$, where $\bar{T}^{*}=\mathscr{F} \backslash \mathscr{H}^{*}$, has maximum estimation capacity. In this case, if a design $T$ has maximum estimation capacity, then $\bar{T}$ must have the structure described above.

PRoof. First of all, the stated condition that any three or four distinct points of $\mathscr{H}^{*}$ are linearly independent is equivalent to that $\mathscr{H}^{*}$ contains no line, and that for any four distinct points $\boldsymbol{\alpha}_{j}, \boldsymbol{\alpha}_{r}, \boldsymbol{\alpha}_{s}, \boldsymbol{\alpha}_{t} \in \mathscr{H}^{*}$, the lines determined by the two pairs $\left(\boldsymbol{\alpha}_{j}, \boldsymbol{\alpha}_{r}\right)$ and $\left(\boldsymbol{\alpha}_{s}, \boldsymbol{\alpha}_{t}\right)$ do not intersect. Each point in $\mathscr{F}$ belongs to $2^{w-1}-1$ lines which are completely inside $\mathscr{F}$; therefore if $\mathscr{H}$ is a $\left(2^{w}-1-f\right)$-subset of $\mathscr{F}$ which contains no line, and $\bar{T}=\mathscr{F} \backslash \mathscr{H}$, then for 
each $1 \leq i \leq f, \phi_{i}(\bar{T})=2^{w-1}-1-\left(2^{w}-1-f\right)+\gamma_{i}(\bar{T})$, where $\gamma_{i}(\bar{T})$ is the number of pairs $\left(\boldsymbol{\alpha}_{j}, \boldsymbol{\alpha}_{r}\right), \boldsymbol{\alpha}_{j}, \boldsymbol{\alpha}_{r} \in \mathscr{H}, j<r$, such that $\boldsymbol{\alpha}_{j}, \boldsymbol{\alpha}_{r}$ and the $i$ th point of $\bar{T}$ are collinear. If for any four distinct points $\boldsymbol{\alpha}_{j}, \boldsymbol{\alpha}_{r}, \boldsymbol{\alpha}_{s}, \boldsymbol{\alpha}_{t} \in \mathscr{H}^{*}$, the lines determined by the two pairs $\left(\boldsymbol{\alpha}_{j}, \boldsymbol{\alpha}_{r}\right)$ and $\left(\boldsymbol{\alpha}_{s}, \boldsymbol{\alpha}_{t}\right)$ do not intersect, then each $\gamma_{i}\left(\bar{T}^{*}\right)$ is either 1 or 0 . Then the $\phi_{i}\left(\bar{T}^{*}\right)$ 's differ from one another by at most 1 . Since $\bar{T}^{*}$ also maximizes $\sum_{i=1}^{f} \phi_{i}(\bar{T})$, it is clear that $\phi\left(\bar{T}^{*}\right)$ is upper weakly majorized by $\boldsymbol{\phi}(\bar{T})$ for all $f$-subsets $\bar{T}$ of $P G(n-k-1,2)$. By Theorem 2.1, the design given by $T^{*}$ has maximum estimation capacity.

If any other $T$ also represents a design with maximum estimation capacity, then $\phi(\bar{T})$ can be obtained from $\phi\left(\bar{T}^{*}\right)$ by permuting its elements. Then $\bar{T}$, like $\bar{T}^{*}$, contains the maximum possible number of lines so that $\bar{T}=\mathscr{F} \backslash \mathscr{H}$, where $\mathscr{F}$ is some $(w-1)$-flat and $\mathscr{H}(\subset \mathscr{F})$ contains no line at all. Also as $\phi(\bar{T})$ is a permutation of $\phi\left(\bar{T}^{*}\right)$, with reference to such $\mathscr{H}$, each $\gamma_{i}(\bar{T})$ is either 1 or 0 , that is, each point of $\bar{T}$ lies on at most one line determined by two points of $\mathscr{H}$. Since $\mathscr{H}$ contains no line, it follows that for any four distinct points $\boldsymbol{\alpha}_{j}, \boldsymbol{\alpha}_{r}, \boldsymbol{\alpha}_{s}, \boldsymbol{\alpha}_{t} \in \mathscr{H}$, the lines determined by the two pairs $\left(\boldsymbol{\alpha}_{j}, \boldsymbol{\alpha}_{r}\right)$ and $\left(\boldsymbol{\alpha}_{s}, \boldsymbol{\alpha}_{t}\right)$ do not intersect.

REMARK. In the situations covered by Theorem 3.1, it is clear that $\operatorname{rank}\left(T^{*}\right)=n-k$ when $w<n-k$. We note that the designs with maximum estimation capacity obtained in Theorem 3.1 have weak minimum aberration and the sizes of the alias sets of two-factor interactions not aliased with main effects are the most uniform possible: they are all equal or differ from one another by at most one.

We now give some applications of Theorem 3.1. For $f=2^{w}-1(2 \leq w<$ $n-k$ ), the design given by $T^{*}$ has maximum estimation capacity if and only if $\bar{T}^{*}$ is a $(w-1)$-flat. For $f=2^{w}-2,2^{w}-3$ or $2^{w}-4(3 \leq w<n-k), T^{*}$ represents a design with maximum estimation capacity if and only if $\bar{T}^{*}$ is obtained by deleting respectively (i) any one point (ii) any two points or (iii) any three noncollinear points from a $(w-1)$-flat. In each of these cases, it is clear that the structure of $\bar{T}^{*}$ is the only one that can maximize $\sum_{i=1}^{f} \phi_{i}(\bar{T})$, and therefore the criteria of minimum aberration, weak minimum aberration and maximum estimation capacity lead to the same optimal design. In the cases of $f=2^{w}-1$ or $2^{w}-2$, under $T^{*}$, all the alias sets of two-factor interactions which are not aliased with main effects are of the same size.

For $f=2^{w}-5(4 \leq w<n-k), T^{*}$ represents a design with maximum estimation capacity if and only if $\bar{T}^{*}$ is obtained by deleting any four linearly independent points from a $(w-1)$-flat. In this case, a design $T$ has weak minimum aberration if and only if $\bar{T}$ is obtained by deleting from a $(w-1)$ flat four points in which any three are noncollinear. Clearly these four points do not have to be linearly independent. However, it is easy to see that $T$ has minimum aberration if and only if the four deleted points are linearly independent. Therefore in this case a design has maximum estimation capacity if and only if it has minimum aberration, but a design with weak 
minimum aberration does not have maximum estimation capacity unless it also has minimum aberration.

For $f=2^{w}-6(4 \leq w \leq n-k)$, the design given by $T^{*}$ has maximum estimation capacity if $\bar{T}^{*}$ is obtained by deleting from a $(w-1)$-flat any five points of the form $\boldsymbol{\alpha}_{j}, \boldsymbol{\alpha}_{r}, \boldsymbol{\alpha}_{s}, \boldsymbol{\alpha}_{t}$, and $\boldsymbol{\alpha}_{j}+\boldsymbol{\alpha}_{r}+\boldsymbol{\alpha}_{s}+\boldsymbol{\alpha}_{t}$, where $\boldsymbol{\alpha}_{j}, \boldsymbol{\alpha}_{r}, \boldsymbol{\alpha}_{s}, \boldsymbol{\alpha}_{t}$ are linearly independent. However, unless $w=4$, this is not the only structure of $\bar{T}$ to ensure maximum estimation capacity. For example, when $w \geq 5$, let $\bar{T}_{1}^{*}$ be obtained by deleting five linearly independent points from a $(w-1)$-flat. Then $T_{1}^{*}$ also gives a design with maximum estimation capacity. It can be seen that this design has minimum aberration. On the other hand, $T^{*}$ does not represent a minimum aberration design unless $w=4$.

Similarly, for $f=2^{w}-7(5 \leq w \leq n-k), T^{*}$ has maximum estimation capacity if $\bar{T}^{*}$ is obtained by deleting from a $(w-1)$-flat six points of the form $\boldsymbol{\alpha}_{j}, \boldsymbol{\alpha}_{r}, \boldsymbol{\alpha}_{s}, \boldsymbol{\alpha}_{t}, \boldsymbol{\alpha}_{u}$ and $\boldsymbol{\alpha}_{j}+\boldsymbol{\alpha}_{r}+\boldsymbol{\alpha}_{s}+\boldsymbol{\alpha}_{t}+\boldsymbol{\alpha}_{u}$, where $\boldsymbol{\alpha}_{j}, \boldsymbol{\alpha}_{r}, \boldsymbol{\alpha}_{s}, \boldsymbol{\alpha}_{t}$, and $\boldsymbol{\alpha}_{u}$ are linearly independent. For $f=2^{w}-8(6 \leq w \leq n-k)$, one choice of $\bar{T}^{*}$ is to delete from a $(w-1)$-flat seven points of the form $\boldsymbol{\alpha}_{j}, \boldsymbol{\alpha}_{r}, \boldsymbol{\alpha}_{s}, \boldsymbol{\alpha}_{t}$, $\boldsymbol{\alpha}_{u}, \boldsymbol{\alpha}_{v}$ and $\boldsymbol{\alpha}_{j}+\boldsymbol{\alpha}_{r}+\boldsymbol{\alpha}_{s}+\boldsymbol{\alpha}_{t}+\boldsymbol{\alpha}_{u}+\boldsymbol{\alpha}_{v}$, where $\boldsymbol{\alpha}_{j}, \boldsymbol{\alpha}_{r}, \boldsymbol{\alpha}_{s}, \boldsymbol{\alpha}_{t}, \boldsymbol{\alpha}_{u}$ and $\boldsymbol{\alpha}_{v}$ are linearly independent, while for $f=2^{w}-9(6 \leq w \leq n-k)$, the eight points $\boldsymbol{\alpha}_{j}, \boldsymbol{\alpha}_{r}, \boldsymbol{\alpha}_{s}, \boldsymbol{\alpha}_{t}, \boldsymbol{\alpha}_{u}, \boldsymbol{\alpha}_{v}, \boldsymbol{\alpha}_{j}+\boldsymbol{\alpha}_{r}+\boldsymbol{\alpha}_{s}+\boldsymbol{\alpha}_{t}$ and $\boldsymbol{\alpha}_{s}+\boldsymbol{\alpha}_{t}+\boldsymbol{\alpha}_{u}+\boldsymbol{\alpha}_{v}$ are to be deleted.

It is clear that in all the cases considered above, except the one corresponding to $f=2^{n-k}-9, n=8, k=1, \operatorname{rank}\left(T^{*}\right)=n-k$.

It follows from the above results that all the 16-run minimum aberration designs with $n \geq 8$ maximize the number of two-factor interactions which are not aliased with main effects, and the sizes of alias sets of such interactions differ from one another by at most one. The same is true for all the 32-run minimum aberration designs with $n \geq 16$, except $n=22$ and 23. For $2^{22-17}$ designs, a minimum aberration design has $\phi\left(\bar{T}_{1}\right)=(1,1,3,3,3,3,3,3,4)$, and there is another design with $\phi\left(\bar{T}_{2}\right)=(2,2,2,2,2,2,3,3,3)$. The elements of $\phi\left(\bar{T}_{1}\right)$ are not so uniform as those of $\phi\left(\bar{T}_{2}\right)$, but $\sum_{i=1}^{f} \phi_{i}\left(\bar{T}_{1}\right)$ is larger than $\sum_{i=1}^{f} \phi_{i}\left(\bar{T}_{2}\right)$. Even though $\phi\left(\bar{T}_{1}\right)$ is not upper weakly majorized by $\phi\left(\bar{T}_{2}\right)$, one can verify directly that $E_{u}\left(T_{1}\right)>E_{u}\left(T_{2}\right)$ for all $u$. A computer search confirms that the $2^{22-17}$ design with minimum aberration has maximum estimation capacity. The same is also true for $2^{23-18}$ designs. Thus for 16-run $2^{n-(n-4)}$ designs with $n \geq 8$ and 32-run $2^{n-(n-5)}$ designs with $n \geq 16$, designs with minimum aberration also have maximum estimation capacity. The situation where $n$ is less than half of the run size (this is also the case where designs with minimum aberration are of resolution at least four) is different. In this case, minimum aberration designs tend not to have the maximum number of nonzero $m_{i}(T)$ 's. As a result, they tend not to maximize $E_{u}(T)$ for large $u$ 's.

4. $s^{n-k}$ designs with maximum estimation capacity. In this section we present some results on $s^{n-k}$ designs. 
LEMma 4.1. Let $\bar{T}$ be any $f(\geq 3)$-subset of $P G(n-k-1, s)$. Then:

(a) For each $1 \leq i \leq f$,

$$
\phi_{i}(\bar{T}) \leq \frac{1}{2}(f-1) \min \{f-2, s-1\}
$$

(b) For $3 \leq f \leq s+1$, equality holds in (4.1) for all $1 \leq i \leq f$ if and only if $\operatorname{rank}(\bar{T})=2$.

(c) For $f>s+1$, equality holds in (4.1) for all $1 \leq i \leq f$ if and only if $f=\left(s^{w}-1\right) /(s-1)$ and $\bar{T}$ is $a(w-1)$-flat, with $3 \leq w \leq n-k$.

Proof. (a) For fixed $\boldsymbol{\alpha} \in \bar{T}$, counting the number of triplets $\{\boldsymbol{\alpha}, \boldsymbol{\beta}, \boldsymbol{\gamma}\}$ such that $\boldsymbol{\alpha}, \boldsymbol{\beta}, \boldsymbol{\gamma}$ are distinct members of $\bar{T}$, we have

$$
\phi_{i}(\bar{T}) \leq \frac{1}{2}(f-1)(f-2) .
$$

Again, for any $\boldsymbol{\alpha}, \boldsymbol{\beta} \in \bar{T}, \boldsymbol{\alpha} \neq \boldsymbol{\beta}$, there are $s-1$ distinct points in $P G(n-k$ $-1, s)$ which are linearly dependent on $\boldsymbol{\alpha}$ and $\boldsymbol{\beta}$, but are different from both of them. Since for distinct $\boldsymbol{\alpha}, \boldsymbol{\beta}, \boldsymbol{\gamma}$, the linear dependence of $\{\boldsymbol{\alpha}, \boldsymbol{\beta}, \boldsymbol{\gamma}\}$ is equivalent to that of $\{\boldsymbol{\alpha}, \boldsymbol{\gamma}, \boldsymbol{\beta}\}$, this yields

$$
\phi_{i}(\bar{T}) \leq \frac{1}{2}(f-1)(s-1) .
$$

Combining (4.2) and (4.3), inequality (4.1) follows.

(b) For $3 \leq f \leq s+1$, (4.1) reduces to (4.2) where equality holds if and only if every three distinct members of $\bar{T}$ are linearly dependent, that is, $\operatorname{rank}(\bar{T})=2$.

(c) For $f>s+1,(4.1)$ reduces to (4.3) where equality holds if and only if $\bar{T}$ is closed, up to proportionality, under the formation of nonnull linear combinations. This happens if and only if the stated conditions hold.

As before, to avoid trivialities, let $f \geq 3$. The case $n-k=1$ is trivial since then $g=\left[\left(s^{n-k}-1\right) /(s-1)\right]$ equals unity. Let $n-k=2$. Then $g=s+1$, $n=k+2 \geq 3$. In this situation, for all $n$-subsets $T$ of $P G(n-k-1, s)$, we have $\operatorname{rank}(\bar{T})=2$ so that by (1.1), Lemma 2.1, Lemma 2.2 and parts (a) and (b) of Lemma 4.1, all regular $s^{n-k}$ resolution $\mathrm{III}^{+}$designs are equivalent with respect to estimation capacity. All such designs are also equivalent under the criterion of minimum aberration.

Turning to the situation $n-k \geq 3$, we have the following consequences of Theorem 2.1 and Lemma 4.1.

THEOREM 4.1. Let $n-k \geq 3$ and $3 \leq f \leq s+1$. Then a regular $s^{n-k}$ resolution $\mathrm{III}^{+}$design $T$ has maximum estimation capacity if and only if $\operatorname{rank}(\bar{T})=2$.

TheOREM 4.2. Let $f=\left(s^{w}-1\right) /(s-1)$ where $3 \leq w<n-k$. Then $a$ regular $s^{n-k}$ resolution $\mathrm{III}^{+}$design $T$ has maximum estimation capacity if and only if $\bar{T}$ is a $(w-1)$-flat. 
Note that the condition $3 \leq w<n-k$ in Theorem 4.2 is not restrictive. This is because (1) if $w=1$, then $f=1$ which is trivial, (2) if $w=2$, then $f=s+1$ which is covered by Theorem 4.1 and (3) if $w=n-k$, then $f=g$, that is, $n=0$ which is impossible. A comparison with Suen, Chen and Wu (1997) shows that under the set-up of Theorems 4.1 or 4.2 , a design has maximum estimation capacity if and only if it has minimum aberration.

REMARK. In the context of Theorems 4.1 and 4.2, designs with minimum aberration maximize $\phi_{i}(\bar{T})$ for each $i$. Therefore to show that these designs have maximum estimation capacity, there is no need to invoke the majorization argument. This is because $E_{u}(T)$ is a monotone increasing function of the $\phi_{i}$ 's. In fact, for two level designs discussed in Section 3, it can be shown that (i) when $f=2^{w}-2, \phi_{i} \leq 2^{w-1}-2$ for all $1 \leq i \leq f$; (ii) when $f=2^{w}-3$, $\phi_{i} \leq 2^{w-1}-2$ for all $i$ and the equality holds for at most one $i$ and (iii) when $f=2^{w}-4, \phi_{i} \leq 2^{w-1}-3$ for all $i$ and the equality holds for at most three $i$ 's. In all these cases, designs with minimum aberration also maximize all the $\phi_{i}$ 's. This is no longer true for $f=2^{w}-a, a \geq 5$. For instance, for $f=2^{w}-5$, a design with minimum aberration has all $\phi_{i} \leq 2^{w-1}-4$, but there are designs with some $\phi_{i}=2^{w-1}-3$.

EXAmPLE 4.1. Let $s=4, n=17, k=14$. Then $g=21, f=4$. Let $\bar{T}$ consist of the points given by the columns of the matrix

$$
\left[\begin{array}{llll}
1 & 0 & 1 & 1 \\
0 & 1 & 1 & y \\
0 & 0 & 0 & 0
\end{array}\right]
$$

where $y$ is a primitive element of $G F(4)$. Arranging the remaining points as columns, we get the matrix

$$
\left[\begin{array}{ccccccccccccccccc}
1 & 1 & 1 & 1 & 1 & 1 & 1 & 1 & 1 & 1 & 1 & 1 & 1 & 0 & 0 & 0 & 0 \\
0 & 0 & 0 & 1 & 1 & 1 & y & y & y & y^{\prime} & y^{\prime} & y^{\prime} & y^{\prime} & 1 & 1 & 1 & 0 \\
1 & y & y^{\prime} & 1 & y & y^{\prime} & 1 & y & y^{\prime} & 0 & 1 & y & y^{\prime} & 1 & y & y^{\prime} & 1
\end{array}\right],
$$

where $y^{\prime}=y+1$. Since $\operatorname{rank}(\bar{T})=2$, by Theorem 4.1 , the $4^{17-14}$ resolution $\mathrm{III}^{+}$design, consisting of level combinations given by the vectors in the row space of the above $3 \times 17$ matrix, has maximum estimation capacity and minimum aberration.

EXAMPLE 4.2. Let $s=3, n=27, k=23$. Then $g=40, f=13\left[=\left(s^{3}-1\right) /\right.$ $(s-1)$ ]. Let $\bar{T}$ be the 2 -flat consisting of the points given by the columns of the matrix

$$
\left[\begin{array}{lllllllllllll}
1 & 0 & 0 & 1 & 1 & 1 & 1 & 0 & 0 & 1 & 1 & 1 & 1 \\
0 & 1 & 0 & 1 & 2 & 0 & 0 & 1 & 1 & 1 & 1 & 2 & 2 \\
0 & 0 & 1 & 0 & 0 & 1 & 2 & 1 & 2 & 1 & 2 & 1 & 2 \\
0 & 0 & 0 & 0 & 0 & 0 & 0 & 0 & 0 & 0 & 0 & 0 & 0
\end{array}\right]
$$


Then by Theorem 4.2 , the resulting $3^{27-23}$ resolution $\mathrm{III}^{+}$design has maximum estimation capacity and minimum aberration.

We now investigate $3^{n-k}$ resolution $\mathrm{III}^{+}$designs involving 27 runs. Then $n-k=3, g=13$ and $4 \leq n \leq 13$. The case $n=13$ is trivial while for $n=11,12$, all designs are isomorphic; see Chen, Sun and Wu (1993). For $4 \leq n \leq 10$, Table 6 of Chen, Sun and Wu (1993) lists all nonisomorphic designs and ranks them with respect to aberration. For each such $n$, we compared all the designs with regard to estimation capacity. Except for only two pairs of designs, the majorization argument given in Theorem 2.1 was successful. For the two exceptional pairs, equation (1.1) had to be applied explicitly. This reveals that in all cases, not only the minimum aberration designs have maximum estimation capacity, but also the two criteria lead to identical ordering of the designs. In other words, for any two designs $T_{1}$ and $T_{2}$, if $T_{1}$ has less aberration than $T_{2}$, then $T_{1}$ also dominates $T_{2}$ in terms of estimation capacity. Consideration of complementary subsets significantly simplifies the computation for large $n$. Incidentally, there are some printing errors in Table 6 of Chen, Sun and Wu. The entries under "additional columns" for their designs 6-3.2 and 6-3.3 should be interchanged; the same thing should be done for their designs 7-4.2 and 7-4.3.

Acknowledgments. We are grateful to the referees and an Associate Editor for their constructive suggestions.

\section{REFERENCES}

Bose, R. C. (1947). Mathematical theory of the symmetrical factorial design. Sankhya 8 107-166. Box, G. E. P. and HunTeR, J. S. (1961). The $2^{k-p}$ fractional factorial designs. Technometrics 3 311-351, 449-458.

Chen, H. and Hedayat, A. S. (1996). $2^{n-m}$ fractional factorial designs with weak minimum aberration. Ann. Statist. 24 2536-2548.

Chen, J. (1992). Some results on $2^{n-k}$ fractional factorial designs and search for minimum aberration designs. Ann. Statist. 20 2124-2141.

Chen, J., Sun, D. X. and Wu, C. F. J. (1993). A catalogue of two-level and three-level fractional factorial designs with small runs. Internat. Statist. Rev. 61 131-145.

Chen, J. and Wu, C. F. J. (1991). Some results on $s^{n-k}$ fractional factorial designs with minimum aberration or optimal moments. Ann. Statist. 19 1028-1041.

Cheng, C. S., Steinberg, D. M. and Sun, D. X. (1998). Minimum aberration and model robustness for two-level factorial designs. J. Roy. Statist. Soc. Ser. B. To appear.

Franklin, M. F. (1984). Constructing tables of minimum aberration $p^{n-m}$ designs. Technometrics 26 225-232.

Fries, A. and Hunter, W. G. (1980). Minimum aberration $2^{k-p}$ designs. Technometrics 22 601-608.

Suen, C.-Y., Chen, H. and Wu, C. F. J. (1997). Some identities on $q^{n-m}$ designs with application to minimum aberration designs. Ann. Statist. 25 1176-1188. 
Sun, D. X. (1993). Estimation capacity and related topics in experimental designs. Ph.D. dissertation, Univ. Waterloo.

TANG, B. and WU, C. F. J. (1996). Characterization of minimum aberration $2^{n-m}$ designs in terms of their complementary designs. Ann. Statist. 24 2549-2559.

UNIVERSITY OF CALIFORNIA

DEPARTMENT OF STATisTics

367 Evans HaLl \#3860

BERKELEY, CALIFORNIA 94720-3860

E-MAIL: Cheng@stat.Berkeley.edu
INDIAN INSTITUTE OF MANAGEMENT

JoKa, Diamond HaRbour ROAD

P.O. Box No. 16757, Alipore Post Office

Calcutta 700027

INDIA

E-MAIL: rmuk1@hotmail.com 